\title{
France-Japan synthesis concept on sodium-cooled fast reactor review of a joint collaborative work
}

\author{
Gilles Rodriguez $^{1, *}$ (D) Frédéric Varaine ${ }^{1}$, Laurent Costes $^{1}$, Christophe Venard ${ }^{1}$, Frédéric Serre ${ }^{1}$, Frédéric \\ Chanteclair $^{1}$, Marie-Sophie Chenaud ${ }^{1}$, Franck. Dechelette ${ }^{1}$, Edouard Hourcade ${ }^{1}$, David Plancq ${ }^{1}$, Jean-Marie Hamy ${ }^{2}$, \\ Jean-François Dirat ${ }^{2}$, Bernard Carluec ${ }^{2}$, Benoit Perrin ${ }^{2}$, Denis Verrier $^{2}$, Shigenobu Kubo ${ }^{3}$, Nobuyuki Ishikawa ${ }^{3}$, \\ Masaaki Tanaka ${ }^{3}$, Kazuya Takano ${ }^{3}$, Shigeo Ohki ${ }^{3}$, Takayuki Ozawa ${ }^{3}$, Hidemasa Yamano ${ }^{3}$, Yoshio Shimakawa ${ }^{4}$, \\ Haruo Mochida ${ }^{4}$, Ryo Shimizu ${ }^{4}$, Shinya Kosaka ${ }^{4}$, Yumi Yamada ${ }^{4}$, Kazuya Koyama ${ }^{4}$, Hisatomo Murakami ${ }^{4}$, \\ Toru Iitsuka ${ }^{4}$, Kazuhiro Oyama ${ }^{4}$, Fumiaki Kaneko ${ }^{4}$, Koichi Higurashi ${ }^{4}$, and Koichi Kurita ${ }^{5}$ \\ ${ }^{1}$ CEA Cadarache DES/IRESNE 13108 Saint-Paul lez Durance Cedex, France \\ 2 Framatome, 10 Rue Juliette Récamier, 69006 Lyon, France \\ 3 JAEA, 4002, Narita, Oarai, Ibaraki, 311-1393, Japan \\ ${ }^{4}$ Mitsubishi FBR Systems Inc., 34-17, Jingumae 2, Shibuya, Tokyo 150-0001, Japan \\ ${ }^{5}$ Mitsubishi Heavy Industries, Ltd., 1-1, Wadasaki 1, Hyogo, Kobe 652-8585, Japan
}

Received: 19 April 2021 / Received in final form: 17 August 2021 / Accepted: 19 August 2021

\begin{abstract}
In the frame of the France-Japan agreement on nuclear collaboration, a bilateral collaboration agreement on nuclear energy was signed on March 21st, 2017, including a topic dedicated to Sodium-cooled Fast Reactor (SFR). This agreement has set the framework to start a bilateral discussion on a joint view of an SFR concept. France (CEA and FRAMATOME) and Japan (JAEA, MHI and MFBR) have carried out these studies from 2017 to 2019. Based on the beginning of the basic design phase of ASTRID project - ASTRID - 600 MWe (ASTRID for Advanced Sodium Technological Reactor for Industrial Demonstration), the two countries performed a common work to examine ways to develop a feasible common design concept, which could be realized both in France and in Japan. The subject was then extended and extrapolated with the ASTRID - 150 MWe data (reduced power reactor and enhanced experimental capabilities) in a second phase of this study. France and Japan first focused on design requirements. Common requirements were identified, as well as differences in the safety approach and the structural design requirements, according to national standards and respective site conditions, in particular considering seismic hazards. The teams developed common Top-Level design Requirements (TLRs) to allow common specification data, then joint design. This collaborative work was carried out through the implementation of twelve France-Japan Working Groups, working jointly. This paper is providing a review of this joint synthesis on Sodium Fast Reactor design concept. It is summarizing the context and objectives, then the definition and approaches of the Top Level Requirements. This paper is then dealing with the major design features: the core design and their related safety aspects, and the nuclear island design. Thus, this paper is providing a comprehensive review of this joint work gathering French and Japan nuclear design teams during two full years.
\end{abstract}

\section{Introduction}

In the frame of the France - Japan agreement on nuclear collaboration, the first collaborative agreement on ASTRID signed in 2014 (May 5th) has been successively conducted [1]. Another France - Japan bilateral collaboration agreement on nuclear energy was further signed in March 21st 2017 [2], including a specific chapter dedicated

\footnotetext{
* e-mail: gilles.rodriguez@cea.fr
}

to ASTRID project. This agreement has set the framework to start a deeper bilateral discussion. Indeed, France (CEA and FRAMATOME) and Japan (JAEA, MHI and MFBR) have carried out studies to establish a common technical view regarding sodium-cooled fast reactor concept, which could be the basis for deeper collaborative work between the two countries.

Based on the 600 MWe ASTRID (named ASTRID600) design corresponding to the beginning of the basic design phase of this project [3], Japan and France performed a common work to examine ways to develop a feasible 
common design concept, which could be built both in France and/or in Japan.

Teams first focused on their respective design requirements and licensing aspects in both countries. Common requirements were identified, as well as differences in the safety demonstration and structural design requirements, according to national standards and respective site conditions (in particular for seismic criteria). The teams then developed some so-called "common Top-Level design Requirements (TLRs)".

The project outcomes evolved from the end of 2017. The new design concept 150 MWe ASTRID (named ASTRID150) was considered, with reduced reactor power and enhanced experimental capabilities [4]. The objectives of this new reactor concept were to demonstrate technology of future large reactors by combining a simulation program, and construction and operation of the reactor itself. ASTRID150 then became the basis for the common studies.

This collaborative work was performed by the implementation of twelve technical Working Groups (WGs). This paper is providing a review of this joint synthesis on Sodium Fast Reactor design concept. It is summarizing the context and objectives, then the definition and approaches of the Top Level Requirements. This paper is then dealing with the major design features: the core design and their related safety aspects, and the nuclear island design. Thus, this paper is providing a comprehensive review of this joint work gathering French and Japan nuclear design teams during two full years.

\section{Genesis of the joint project and its organisation}

This agreement settled in 2017 [2] has set the framework to start a deeper bilateral discussion. As specified in the official text of this agreement: "... This discussion aims at: - deepening their exchanges in order to define more clearly a common technical design of the ASTRID demonstrator, especially the means to integrate adjustments of French and Japanese technology to the current design study carried out since 2010.

- identifying a potential new collaboration framework relating to the know-how and experience of both partners, ensuring a fair and appropriate management of the Intellectual Property and taking into account the reflection carried out by the French part on a new organization for the next coming steps of this project.

- identifying in France and in Japan, facilities that shall be used to proceed to validation of the design and $R \& D$ works.

Both partners will make all their possible efforts to achieve the discussion in order to settle the new phase of their collaboration".

To provide a technical organization to this request, all involved parties have initiated a set of three technical Face To Face meetings (from March to July 2017). Then they concluded to organize specific working groups to perform a first step of results, to be presented to a strategic board, at
Table 1. Table of the corresponding Working Group.

\begin{tabular}{|c|l|r|l|}
\hline Group N $^{\circ}$ & \multicolumn{1}{|c|}{ Subject Name } & Group $\mathbf{N}^{\circ}$ & \multicolumn{1}{|c|}{ Subject Name } \\
\hline WG01 & Top Level Requirements & WG07 & Decay Heat Removal System \\
\hline WG02 & Core \& Fuel & WG08 & Fuel Handling System \\
\hline WG03 & Reactor Shutdown & WG09 & Containment \\
\hline WG04 & Severe Accident Mitigation & WG10 & System Consistency \& Cost \\
\hline WG05 & $\begin{array}{l}\text { Reactor \& Primary Heat } \\
\text { Tranport System }\end{array}$ & WG11 & Technological Qualification \\
\hline WG06 & Secondary / tertiary systems & WG12 & Numerical Simulation \\
\hline
\end{tabular}

the end of 2017. The organization has been performed in two steps:

- it started with a series of Face to Face meetings carried out during first semester 2017 to explain the fundamental ASTRID based specifications, the Japanese requirements and the technical points to be converged and those to be pursued for exchange and mutual understanding.

- according to this first step, twelve Working Groups have been created to achieve a better technical common view for an SFR common design reactor.

These working groups have the ability to exchange data and information under the ruling of the France-Japan collaborative arrangement signed in 2014 (implementing arrangement on the ASTRID program and sodium fast reactor).

\section{Main items treated by the working groups and roadmap}

The twelve Working Groups are shown in Table 1. Each of these working groups (except WG10 which operates as a Steering Committee group) had to respect some rules:

- they were composed of a board of specialists nominated by CEA, FRAMATOME, JAEA, MHI and MFBR.

- they have to initiate their actions with a Workplan accepted by all parties.

- they have to perform a regular Progress of Work report to WG01, some interim written synthesis and a final synthesis at the end of 2019.

- the WG01 has to provide consistency between all the other working groups, and to manage data transfer and interfaces amongst the Working Groups. It has to report to the Steering Committee (WG10) the progress status.

The roadmap of this Joint work is resumed as follow: - July 2017: definition of the WG list, organization and technical correspondents and experts.

- August 2017: starting of the first meetings of the Working Groups.

- December 2017: interim report of all WG activities issued to the WG10 Steering Committee.

- January-March 2018: transition phase passing from ASTRID600 to ASTRID150 according to the French new program orientation. WG11 and WG12 are created consequently.

- April 2018-December 2019: continuation of the work to achieve a shared vision of an SFR design reactor $[5,6]$. 


\section{Definition of the top level requirements}

The agreement on TLRs was considered as the basis of the mutual understanding before going further towards the definition of the SFR common reactor design. These TLRs will become the basis for specific design requirements and design conditions applied on WG02 to 09.

The purpose is to seek common agreement of these TLRs and conditions to derive benefits of Japan and France cooperation and the common design. Licensability conditions and Technology Readiness Level applied in every system evaluated by the Working Groups have also to be included within these requirements. In addition, site conditions for earthquake (which have significant impact on final design) have been compared between Japan and France and were reflected in the design requirements.

The resulting work provided two levels of requirement: High Level Requirement, and ASTRID design requirement. Thus, corresponding to two different TLR tables. The first one identified as "General Top Level Requirement \& Targets of demonstration SFR". The second one identified as the "Current ASTRID 600 Top Level Requirement".

The first table is composed of 22 items (see Tab. 2). The TLRs had to fulfill the following items classified in several categories:

- fundamental Design Specifications.

- strategic requirements defining the strategy related to the industrial size SFR definition.

- safety requirements related to licensing and the protection of public and staff.

- economical \& Performances requirements including investment issues and operational effectiveness issues.

- sustainability \& Environment requirements.

- proliferation Resistance \& Physical Protection requirements.

- codes and standards.

The second table started from the ASTRID600 key requirements table at its Basic Design phase (2017). It was then adjusted by both partners to be consistent with the first table (General TLRs \& Targets of demonstration SFR), and also to comply with specific Japanese requirements. This second table is made of 21 major requirements subdivided in 43 requirements (or input data), which provides quantified values to be respected by the common team designer.

These two Top Level Requirements Tables were a help to start the work program of the other WGs. In parallel, the WGs from 02 to 09 could define their own specific input data, but they had to make a back-up verification to be consistent with these TLR Tables. Every line of the TLR tables were discussed jointly and their rationale were justified.

\section{From ASTRID 600 to ASTRID 150}

At the end of 2017, the energy context in France changed, which affected the ASTRID600 project. The need for commercial deployment of fast neutron reactors was postponed in France (re-scheduled around the second half of the 21st century). Therefore, CEA proposed a global Fast Neutron Reactor Simulation Program, including experimental facilities, including a demonstration reactor in the range of 100-200 MWe. The High-level objectives of the Sodium Fast neutron Reactor Simulation Program remain the same: it must maintain over the long term, the strategy of the closed fuel cycle, and maintain the capability to deploy a safe, performing and competitive industrial fleet of large size SFR. In this context, the economy approach have to take into account the electricity production aspect, but the fuel cycle and fissile material, $\mathrm{Pu}$ and $\mathrm{U}$ management aspects too.

This new work program will allow developing validated numerical simulation tools that will ease to speed-up design and licensing phases of future commercial SFR (in the range of $1 \mathrm{GWe}$ ) thanks to the combination of experimental program and the feedback experience from the operation of past SFR reactors. In France, the target date to obtain these data is postponed according to the new date for a decision in France to invest into a commercial SFR in the second half of this century. In Japan, according to the Strategic Roadmap for fast reactor development, decided by the nuclear-related ministerial meeting of Japan on 21st December 2018, the fast reactors will be deployed in latter half of 21st century, whereas it is expected that the next fast reactor will start its operation around mid-21st century.

It was then confirmed that the current design work for the 600 MWe ASTRID (named ASTRID600) will be closed by the end of March 2018, and that some new works will be defined after embodiment of the design concept of New ASTRID (also named ASTRID150) from second semester 2018. The downsizing of ASTRID reactor project was accompanied with a Design to Cost approach which aims to re-challenge every option and system of the reactor to try to significantly decrease the investment cost.

CEA has expressed to Japanese partners its willingness to pursue the common design study, moving from ASTRID600 to ASTRID150 project. Technical conditions for this transition phase were evaluated in all the Working Groups. The major question arisen from the Japanese parts was the justification regarding the scaling factor and the way to extrapolate systems and components from a power of 150 MWe (ASTRID150) to a commercial size reactor (in a range from 1 to $1.5 \mathrm{GWe}$ ) without the intermediate scale of ASTRID600.

With the downsizing of ASTRID project down to 150 MWe, the exercise of the demonstration capability to the commercial size reactors was concerned. The extrapolation from 150 MWe up to commercial size reactor was investigated (see Fig. 1) in every item with the view point of:

- the Technical Readiness Level evaluation of all selected options.

- the experimental feedback of previous SFR reactors (Phénix, SUPERPHENIX, MONJU and JOYO reactors). - the potentiality to realize complementary experimental testings on sodium facilities (subject devoted to WG11). 


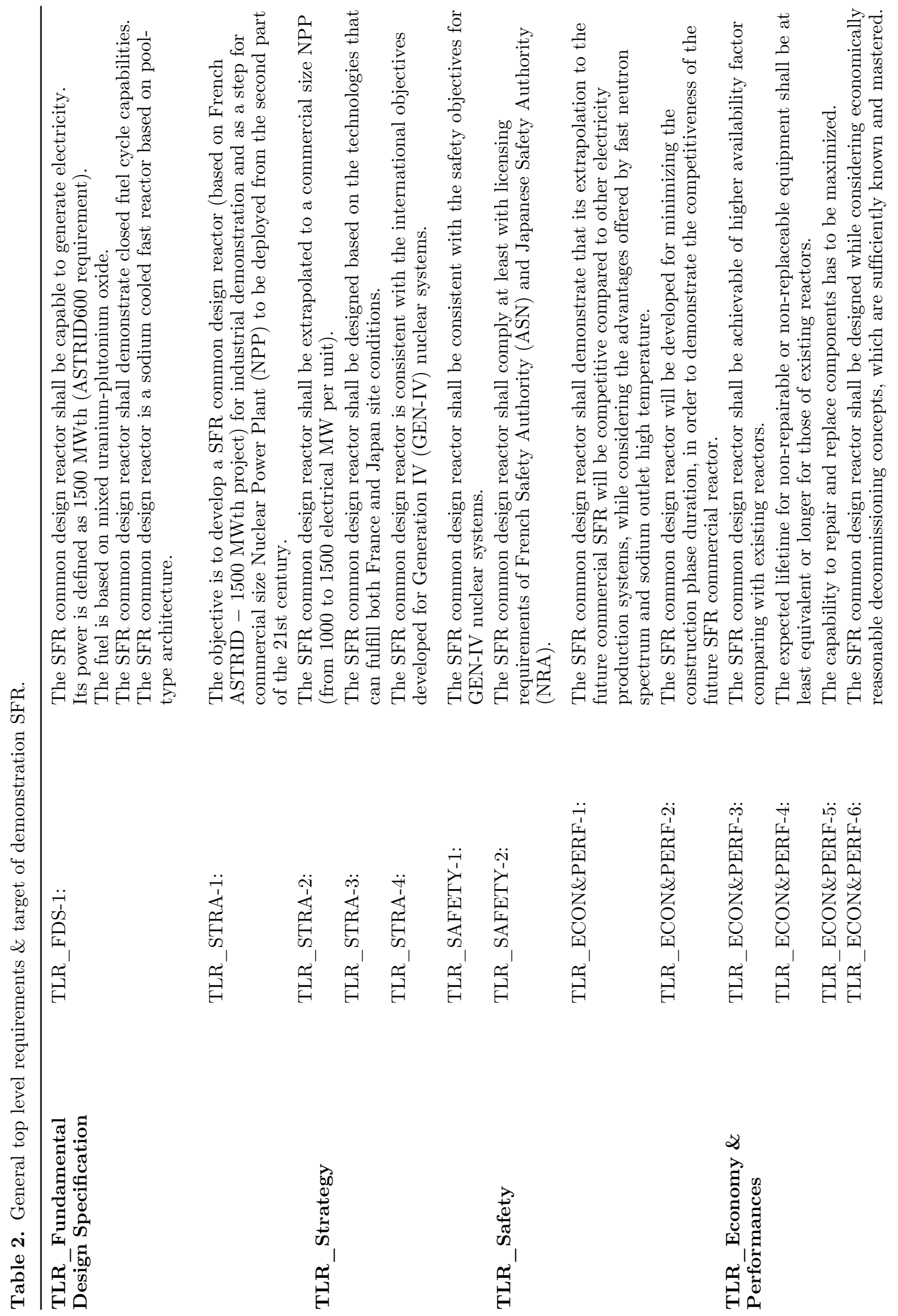



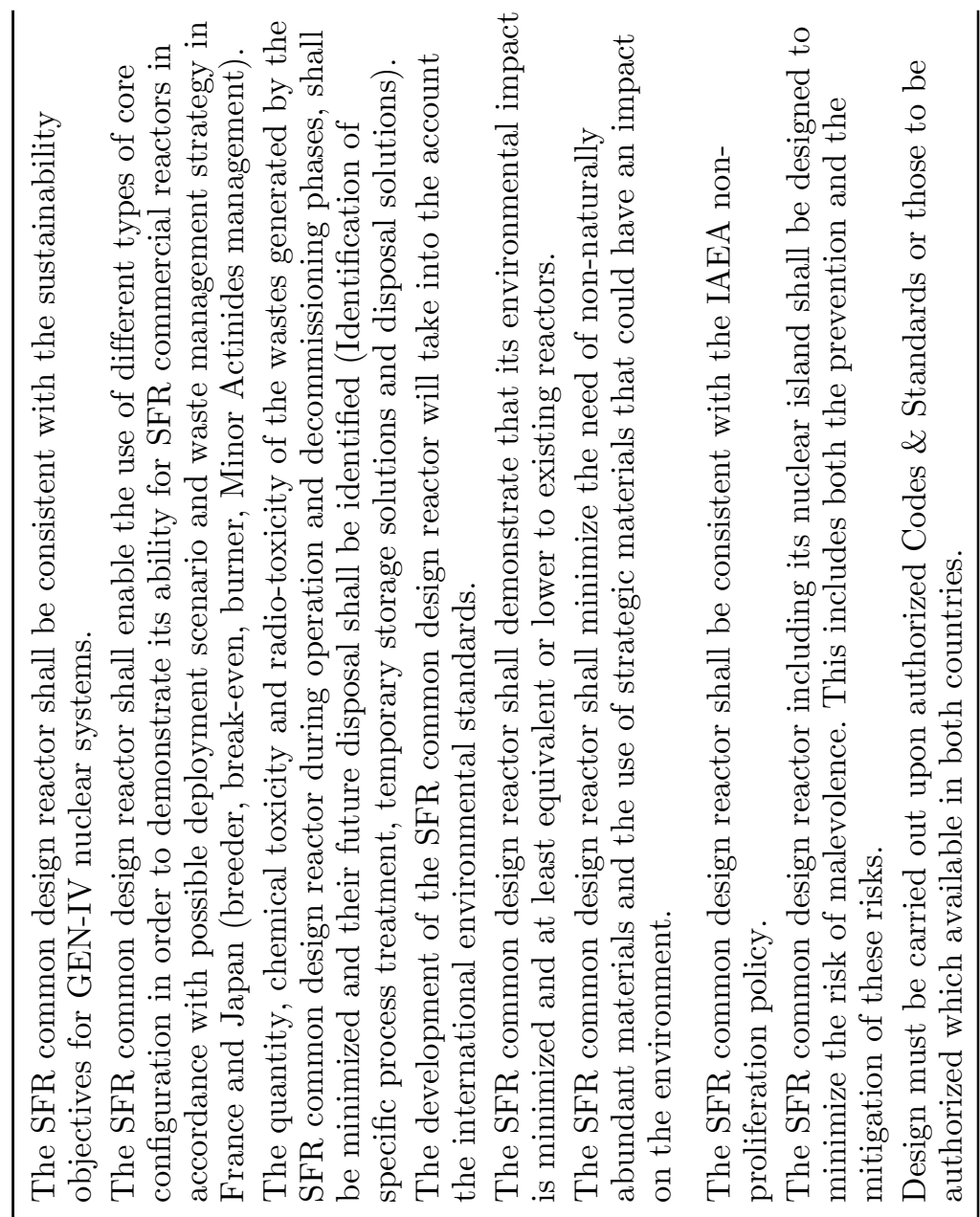

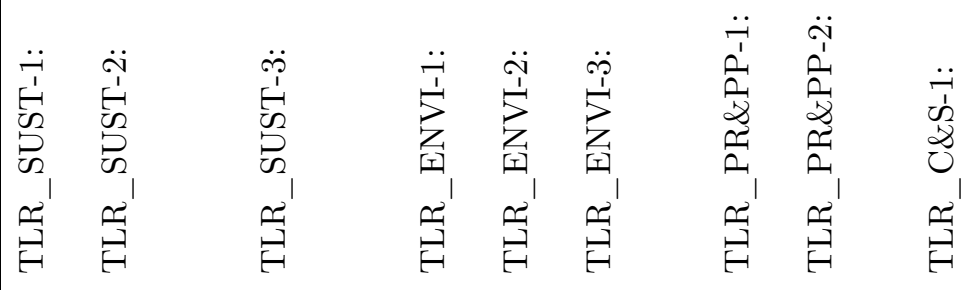

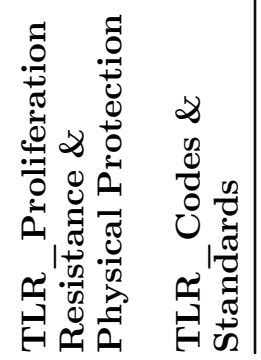




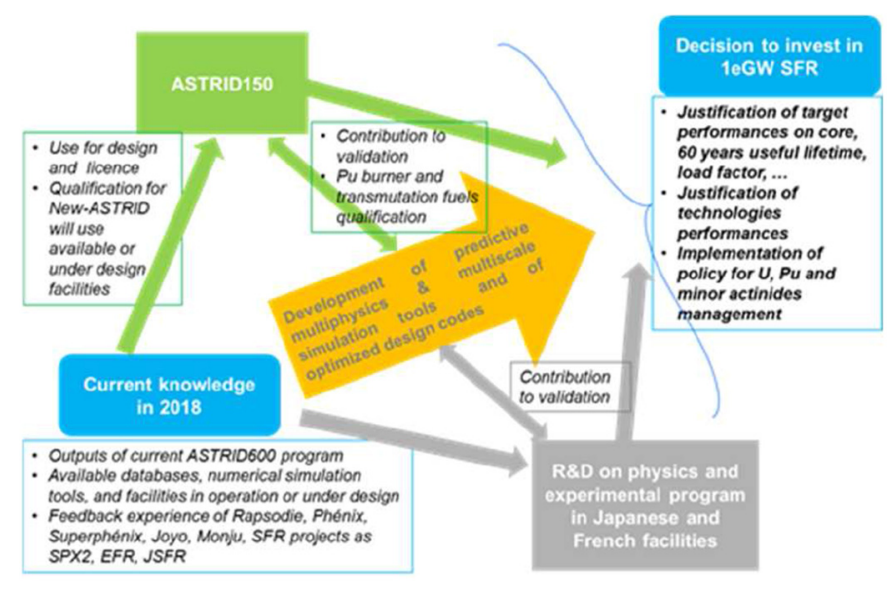

Fig. 1. Objectives of a combined ASTRID150 and a simulation program for scaling up to a commercial size reactor.

- the potentiality of numerical simulation and computing codes to supply some technological gaps thank to modelling (subject devoted to WG12).

- if requested, the scaling up from ASTRID150 to the design of a large scale Sodium Fast Reactor will be confirmed under after a thorough review of the manufacturing capability of all key components, with the help of a Manufacturing Readiness Level evaluation. This last evaluation will be the crucial one that will determine the level of confidence of this scaling up.

\section{Common view related to the top level requirements}

For the Common design concept, it was stated that:

- the SFR common design reactor shall be designed based on the technologies, which can fulfill both France and Japan site conditions.

- ASTRID600 technology is carried out in coherence with the French site conditions, especially regarding seismic conditions. The SFR common design reactor must be designed by minimizing changes on current ASTRID600 design.

- for the SFR common design reactor, the technology selected (in particular regarding anti-seismic protection system) has to be designed in a view of being built in France and Japan by minimizing both additional major research and development due to specific site conditions (see Fig. 2).

At the end, the common design specifications based on ASTRID600 were the following:

- MOX fuel Sodium-cooled Fast Reactor based on pooltype architecture.

- Output power 1500MWth.

- Cooling system: four Intermediate Heat Exchangers (IHX), three Primary Pumps, four secondary loops.

- Core and fuel: CFV type core [7]; (CFV for - in French Coeur à Faible coefficient de Vidange, or Low Void fraction Core in English).

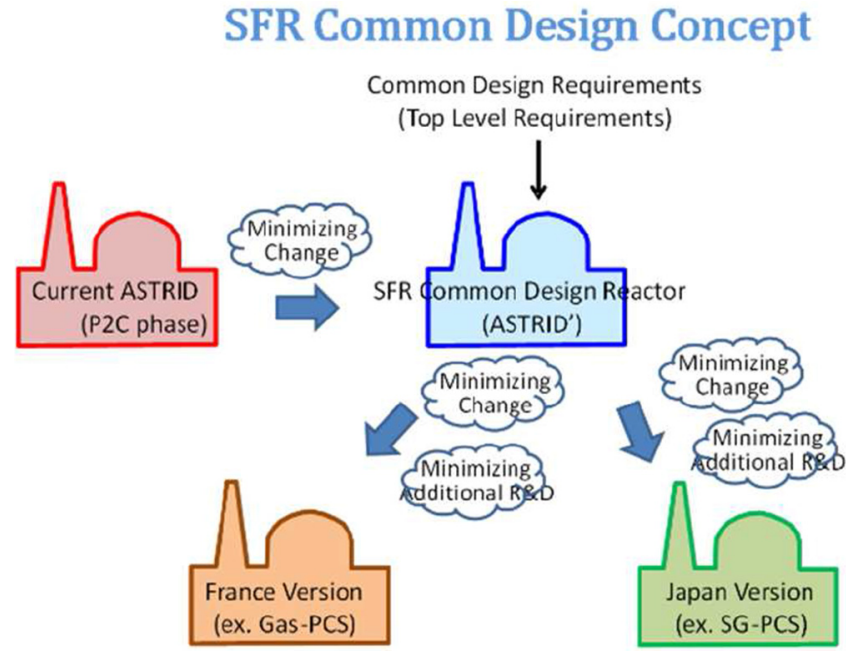

Fig. 2. SFR Common design approach.

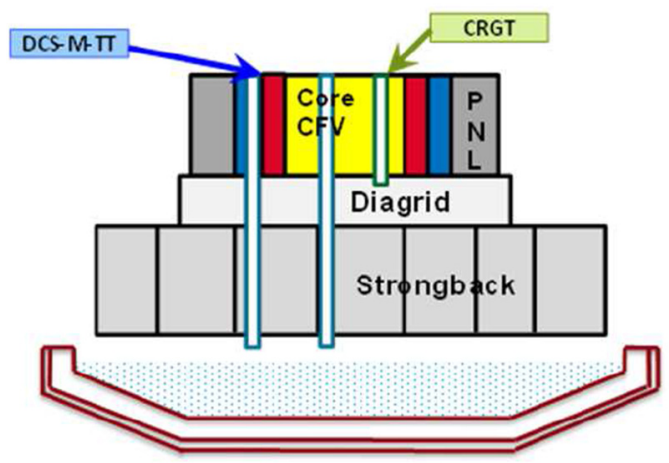

Fig. 3. Schematics of mitigation measures on ASTRID600 (DCS-M-TT: mitigation type safety device to guide the melted core on the core catcher; CRGT: Control Rod Guide Tube; PNL: Lateral Neutronic Protection).

- Reactor shutdown system: two active shutdown systems plus a complementary one (passive).

- Mitigation of core damage: CFV type core plus Transfer Tubes to make a direct discharge of the corium in a large Core catcher (see Fig. 3), strongback design including a Sacrificial box.

- Reactor structure: a Pool-type primary vessel [8] (hanged reactor vessel, simplified plenum separator, bottom supported strong back and diagrid) with High Frequency Design is applicable.

- Secondary plus tertiary systems: steam Power Conversion System (PCS) with four helical coil Steam Generators (SG) or Brayton Gas $\left(\mathrm{N}_{2}\right)$ Power Conversion System [9] with four Sodium Gas heat Exchangers.

- Decay Heat Removal (DHR) system: diverse and redundant systems with natural circulation capability, i.e., Active DHR, Passive DHR and, External vessel cooling and water cooling at the SG.

- Fuel Handling System with limited Internal Vessel Storage (IVS) or with an External In-sodium Buffer Zone (EBZ), and a cask car transportation as the main reference. 
- Need for a Containment Reactor Building plus a Polar Table close to the upper vessel closure.

The common design specification based on ASTRID150 were:

- MOX fuel Sodium-cooled Fast Reactor based on pooltype architecture.

- Output power 400MWth.

- Cooling system: two IHXs, two Primary Pumps, one unique secondary loop and one SG.

- Core and fuel: homogeneous with upper sodium plenum.

- Reactor Shutdown System: two active shutdown systems plus a complementary one (passive).

- Mitigation of core damage: Transfer Tubes with Core catcher and a Sacrificial box.

- Reactor structure: a Pool-type (hanged reactor vessel, simplified plenum separator, bottom supported strong back and diagrid) including High Frequency Design is applicable.

- Secondary plus tertiary systems: steam Power Conversion System with a single helical coil Steam Generator.

- Decay heat removal system: diverse and redundant system with diversified capabilities i.e., Passive DHR plus on External primary vessel cooling and water cooling at the SG.

- Fuel Handling System: IVS and cask car transportation.

- Containment: Reactor Building with Steel Concrete structure without Polar Table.

\section{Major design features: the core design and their related safety aspects}

Japan has investigated the feasibility of enhanced high burnup core and fuel using Oxide Dispersion-Strengthened steel cladding on ASTRID600, considering Japanese core performance targets. In case of applying the French safety requirements and partly relaxed design targets, it has been clarified that the high burn-up low-void-effect (CFV) core is feasible in Japan.

Regarding passive shutdown capabilities, a preliminary analysis has been performed for ASTRID600 regarding Self-Actuated Shutdown System (SASS), that is a Complementary Safety Device (CSD) in Japanese safety approach, in place of a RBH device (an hydraulically suspended absorber rod, that is the dedicated CSD in the current French safety approach) in order to investigate its potential as common design. The preliminary analysis showed good confidence on the ability of SASS to satisfy main requirements.

The mitigation measures of ASTRID600 against severe accidents, such as core catcher and molten corium discharge assembly, as well as the sodium void reactivity features of the CFV core, were appreciated to be prospective for IVR (In-Vessel Retention) strategy in both countries (see Fig. 3).

For ASTRID150 with a reduced reactor power and enhanced experimental capabilities, specific items have been identified to achieve qualification data necessary for the future commercial SFR according to French and Japanese strategies on core \& safety.

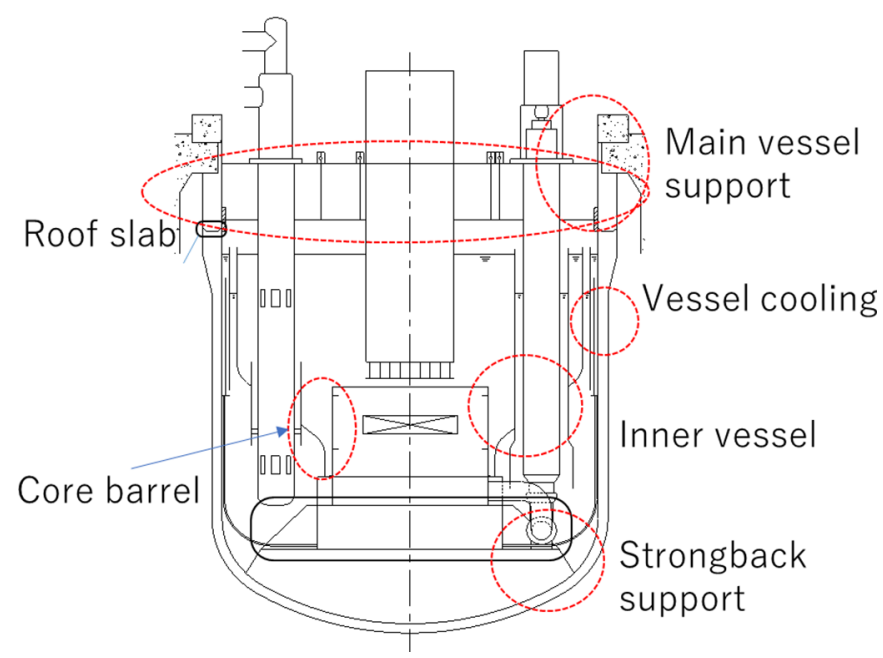

Fig. 4. Discussion parts to improve structural intactness.

\section{Major design features: the nuclear island design}

One of important issues on a reactor structure design is to enhance seismic resistance to be tolerable against strong earthquake such that postulated in Japan. A concept of High Frequency Design (HFD) is shared, in which the natural frequency of the reactor structure should be higher than that of peak acceleration of vertical floor seismic response, and not of the horizontal or combined response. The design options related to HFD have been examined and design recommendations are established (see Fig. 4).

ASTRID600 adopted gas Power Conversion System to strictly eliminate the chemical reaction risks due to the proximity of sodium and water in the steam generator units. On the other hand, Steam Generator (SG) is thought to be a concept with high technical readiness level and is a reference option in Japan and a backup option in France. Then, design comparison of single monolithic SG with single-walled helical coil tube was mainly conducted in this study from the viewpoint of safety, economy and technological maturity.

Common concept of a decay heat removal system is discussed to achieve practical elimination of loss of decay heat removal function.

Fuel handling system studies are performed to eliminate ex-vessel storage of spent fuels in sodium to reduce the construction cost.

And finally, an adequate confinement system is investigated to achieve practical mitigation of large radiological release to the environment even under the condition of core destructive accident.

\section{Path forward and conclusions}

Through fruitful exchanges, France and Japan have obtained good foundation of common objectives, TLRs, and design orientations when dealing with SFR concept. 


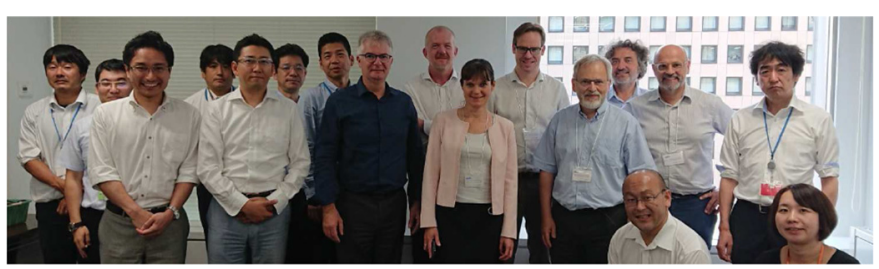

Fig. 5. Most of the whole team members during the final report wrap-up in Tokyo JAEA offices.

France and Japan engineers have reached a good level of mutual understanding in terms of key SFR technologies, design orientations and $R \& D$ needs. These proved that France-Japan collaboration is very beneficial. The achievements obtained will be a strong basis for further R\&D collaboration derived from the technological gaps. This common collaborative R\&D activity is already programmed for the years to come [10]. This France-Japan joint team (see Fig. 5) initiated the reference common vision in terms of future SFR concepts.

Many people were involved in the joint project and it is a good opportunity to thank them for the quality of the work produced and their involvement in this innovative project. The project team is very grateful to all engineers, researchers, SFR specialists and experts coming from CEA, FRAMATOME, JAEA, MFBR, MHI without whom all this work could not be presented.

This study includes some of the results of the "Technical development program on a commercialized FBR plant" and "Technical development program on a fast reactor international cooperation, etc". ensured to JAEA by the Ministry of Economy, Trade and Industry in Japan (METI). We appreciate the valuable advice from the people involved.

\section{Author contribution statement}

The related authors are the main contributors of to the corresponding Working Group: Gilles Rodriguez: WG01, 06, 11 \& 10, Frédéric Varaine: WG01, 10 \& 12, Laurent Costes: WG03 \& 04, Christophe Venard: WG02, Frédéric Serre: WG04, Frédéric Chanteclair: WG09, Marie-Sophie Chenaud: WG05, Franck. Dechelette: WG08, Edouard Hourcade: WG07, David Plancq: WG06, Jean-Marie Hamy: WG01, 06, 11 \& 10, Jean-François Dirat: WG03 \& 05, Bernard Carluec: WG03 \& 04, Benoit Perrin: GW06
\& 07 \& 8, Denis Verrier: WG02, Shigenobu Kubo: WG01, 10 \& 12, Nobuyuki Ishikawa: WG11, Masaaki Tanaka: WG12, Kazuya Takano: WG01, Shigeo Ohki: WG02, Takayuki Ozawa; WG01, Hidemasa Yamano: WG03, Yoshio Shimakawa: WG01, Haruo Mochida: WG01 \& 10, Ryo Shimizu: WG11, Shinya Kosaka: WG12, Yumi Yamada, Kazuya Koyama: WG03, Hisatomo Murakami: WG05, Toru Iitsuka: WG06, Kazuhiro Oyama: WG07, Fumiaki Kaneko: WG08, Koichi Higurashi: WG09, Koichi Kurita: WG01.

\section{References}

1. J. Rouault et al., Japan-France Collaboration on the ASTRID program and Sodium Fast Reactor, in Proc. of the ICAPP 2015 Conference, Nice, France (2015)

2. F. Varaine et al., The significant collaboration of Japan and France on the design of ASTRID Sodium Fast Reactor since 2014, in Proc. of the GIF SYMPOSIUM, Paris, France (2018)

3. F. Varaine et al., ASTRID project, General overview and status progress, in Proc. of the ICAPP 2018 Conference, Charlotte, North Carolina, USA (2018)

4. C. Doderlein et al., Contribution of the ASTRID reactor to the development of the next generation of industrial sodiumcooled Fast Reactors, in Proc. of the ICAPP 2019 Conference, Juan les Pins, France (2019)

5. K. Takano et al., Core and safety design for France-Japan common concept on Sodium-cooled Fast Reactor, Nucl. Eng. Des. J. (2021)

6. A. Kato et al., Plant system study of France-Japan common concept on Sodium-cooled Fast Reactor, Nucl. Eng. Des. J. (2021)

7. C. Venard et al., The ASTRID core at the midterm of the conceptual design phase, in Proc. of the ICAPP 2015 Conference, Nice, France (2015)

8. M.S. Chenaud et al., Progress in the design of the ASTRID Nuclear Island, in Proc. of the ICAPP 2018 Conference, Charlotte, North Carolina, USA (2018)

9. D. Plancq et al., Status of the ASTRID Gas Power Conversion System option, in Proc. of the GIF SYMPOSIUM, Paris, France (2018)

10. J.C. Garnier et al., Ten years of Japanese and French Research and Industry Collaboration on GENIV-SFR Development: Outcomes and Prospects, in Proc of IAEA International Conference on Fast Reactors and related Fuel Cycles FR22 (CN-291), Beijing, China (2022)

Cite this article as: Gilles Rodriguez, Frédéric Varaine, Laurent Costes, Christophe Venard, Frédéric Serre, Frédéric Chanteclair, Marie-Sophie Chenaud, Franck. Dechelette, Edouard Hourcade, David Plancq, Jean-Marie Hamy, Jean-François Dirat, Bernard Carluec, Benoit Perrin, Denis Verrier, Shigenobu Kubo, Nobuyuki Ishikawa, Masaaki Tanaka, Kazuya Takano, Shigeo Ohki, Takayuki Ozawa, Hidemasa Yamano, Yoshio Shimakawa, Haruo Mochida, Ryo Shimizu, Shinya Kosaka, Yumi Yamada, Kazuya Koyama, Hisatomo Murakami, Toru Iitsuka, Kazuhiro Oyama, Fumiaki Kaneko, Koichi Higurashi, Koichi Kurita, France--Japan synthesis concept on sodium-cooled fast reactor review of a joint collaborative work, EPJ Nuclear Sci. Technol. 7, 15 (2021) 